\title{
REIC/Dkk-3-encoding adenoviral vector as a potentially effective therapeutic agent for bladder cancer
}

\author{
TAKESHI HIRATA ${ }^{1}$, MASAMI WATANABE ${ }^{1-3}$, HARUKI KAKU $^{1-3}$, YASUYUKI KOBAYASHI ${ }^{1}$, \\ HIROSHI YAMADA ${ }^{4}$, MASAKIYO SAKAGUCHI ${ }^{5}$, KOHJI TAKEI $^{4}$, \\ NAM-HO HUH ${ }^{5}$, YASUTOMO NASU ${ }^{1-3}$ and HIROMI KUMON ${ }^{1-3}$ \\ ${ }^{1}$ Department of Urology, ${ }^{2}$ Center for Gene and Cell Therapy, ${ }^{3}$ Innovation Center Okayama for \\ Nanobio-Targeted Therapy, Departments of ${ }^{4}$ Neuroscience and ${ }^{5}$ Cell Biology, \\ Graduate School of Medicine, Dentistry and Pharmaceutical Sciences, \\ Okayama University, Okayama, Japan
}

Received January 13, 2012; Accepted March 21, 2012

DOI: 10.3892/ijo.2012.1503

\begin{abstract}
Bladder cancer is one of the most common urogenital malignancies. The intravesical instillation of anticancer agents is an attractive strategy to treat a superficial lesion or floating/disseminated cancer cells after transurethral operation. An adenovirus carrying REIC/Dkk-3, a tumor suppressor gene (Ad-REIC), exhibits cancer-specific apoptotic effects in various types of cancer cells. The aim of the present study was to examine the potential of Ad-REIC as a therapeutic agent for bladder cancer. KK47 and RT4 human bladder cancer cells were sensitive to the Ad-REIC treatment for apoptosis induction, but some human bladder cancer cell lines (T24, J82 and TccSup) were resistant. Significant cell growth inhibition was observed when these resistant cancer cell lines were treated with Ad-REIC in a condition of floating cells, which is clinically observed after transurethral operation and becomes a cause of intravesical cancer dissemination. The therapeutic potential of Ad-REIC for the treatment of multidrug-resistant bladder cancer was investigated. The adriamycin-resistant KK47 bladder cancer cells (KK47/ADM), which also present multidrug resistance, showed induction of significant apoptosis following Ad-REIC treatment. The Ad-REIC treatment induced downregulation of P-glycoprotein in KK47/ADM cells and restored the sensitivity to doxorubicin (adriamycin). Ad-REIC suppressed P-glycoprotein expression in a c-JunNH2-kinase (JNK)-dependent manner. Therefore, the current study indicated two therapeutic aspects of the Ad-REIC agent
\end{abstract}

Correspondence to: Dr Masami Watanabe, Center for Gene and Cell Therapy, Graduate School of Medicine, Dentistry and Pharmaceutical Sciences, Okayama University, 2-5-1 Shikata-cho, Okayama 700-8558, Japan

E-mail: mwcorrespondence@gmail.com

Key words: reduced expression in immortalized cells/Dickkopf-3, bladder cancer, apoptosis, P-glycoprotein, c-Jun-NH2-kinase against human bladder cancer cells, as an apoptosis inducer/ cell growth inhibitor and as a sensitizer of chemotherapeutic agents in multidrug-resistant cancer cells. The intravesical instillation of Ad-REIC could be an attractive therapeutic method in human bladder cancer where the treatment of superficial lesions and floating/disseminated or multidrug-resistant cancer cells is necessary.

\section{Introduction}

Bladder cancer is one of the most common urogenital malignancies. More than 2/3 of bladder cancer presents as superficial (pTa or pT1), which means the tumor is confined to the epithelium or lamina propria. Noninvasive bladder cancer can be completely resected by transurethral resection of bladder tumors. However, $50-70 \%$ of bladder cancer recurs and $15-30 \%$ progresses to muscle invasive disease, despite a complete transurethral operation (1). Therefore, bladder cancer is a highly recurrent disease and intravesical instillation of anti-cancer agents after transurethral operation is an attractive strategy to prevent the cancer cell dissemination and recurrence. Intravesical chemotherapy and/or immunotherapy are often added after a transurethral operation to prevent intravesical cancer recurrence and progression. However, the tumor recurrence rates are still high and reported to be $30-44 \%$ with the adjuvant intravesical treatment (2). Therefore, novel therapeutic agents for the treatment of superficial lesion and floating/disseminated cancer cells are necessary.

The reduced expression in immortalized cell (REIC) gene is identical to Dickkopf-3 (Dkk-3), which is a member of the Dickkopf gene family. Expression of REIC/Dkk-3 gene is significantly downregulated in a broad range of human cancer cells, but typically expressed in non-malignant cells (3-10). The REIC/Dkk-3 is thought to be a tumor suppressor gene and to provide a possible means of gene therapy for human malignant tumors. An adenovirus vector carrying REIC/Dkk-3 (Ad-REIC) induces apoptosis in prostate cancer and testicular cancer, but not in normal cells $(5,9,10)$. Overexpression of REIC/Dkk-3 protein in cancer cells by Ad-REIC treatment leads to endoplasmic 
reticulum (ER) stress and activation of c-Jun-NH2-kinase (JNK), which induce cancer specific cell apoptosis (9).

The aim of present study was to investigate the potential of Ad-REIC as a therapeutic agent for bladder cancer. Recent study showed that some human bladder cancer cell lines are resistant to Ad-REIC treatment for apoptosis induction (11). This study used an in vitro cancer cell floating condition to assess the possibility of Ad-REIC intravesical treatment and re-evaluated the efficacy of Ad-REIC in these resistant bladder cancer cell lines. In addition, the appearance of cancer cells resistant to multiple chemotherapeutic agents is a serious obstacle. Doxorubicin (adriamycin) is a major intravesical chemotherapeutic agent that is used immediately after a transurethral operation to prevent cancer cell dissemination (12). However, doxorubicin resistant bladder cancer has become a clinical problem. The multidrug resistance phenotype is often associated with increased expression of adenosine triphosphate (ATP) binding cassette $(\mathrm{ABC})$ superfamily proteins, $\mathrm{P}$-glycoprotein (P-gp) and multidrug resistance-associated protein1 (MRP1) $(13,14)$. The enhancement of the JNK pathway downregulates P-gp and reverses P-gp mediated multidrug resistance in cancer cells (15). These findings led to the hypothesis that the JNK activation by Ad-REIC treatment might be able to downregulate P-gp and overcome multidrug resistance. Therefore, this study also investigated the potential of Ad-REIC as a sensitizer of chemotherapeutic agents in the adriamycin resistant KK47 bladder cancer cells.

\section{Materials and methods}

Cells and cell culture. Human bladder cancer cell lines, KK47 (KK47/Wt), RT4, T24, J82 and TccSup were obtained from the American Type Culture Collection (Rockville, MD, USA). The adriamycin resistant human bladder cancer cell line KK47/ ADM was kindly provided by Professor S. Naito (Department of Urology, University of Kyushu, Fukuoka, Japan) (16). KK47/ ADM cells were grown in RPMI-1640 medium (Sigma, St. Louis, MO, USA) supplemented with $10 \%$ (v/v) fetal bovine serum, penicillin $(100 \mathrm{IU} / \mathrm{ml})$, streptomycin $(100 \mu \mathrm{g} / \mathrm{ml})$, and $1 \mu \mathrm{M}$ doxorubicin (Adriacin ${ }^{\mathrm{TM}}$, Kyowa Hakkoh Co., Tokyo, Japan). Other bladder cell lines were grown in RPMI-1640 medium supplemented with $10 \%(\mathrm{v} / \mathrm{v})$ fetal bovine serum, penicillin (100 IU/ml) and streptomycin $(100 \mu \mathrm{g} / \mathrm{ml})$.

Adenovirus vector carrying REIC/Dkk-3 (Ad-REIC). A fulllength cDNA of REIC/Dkk-3 was integrated into the cosmid vector, pAxCAwt, and then it was transferred into an adenovirus vector by the COS-TPC method (Takara Bio, Shiga, Japan) (17). An adenovirus vector carrying the LacZ gene (Ad-LacZ) was used as a control, as described previously (5).

Apoptosis assay. A sample of $5.0 \times 10^{5}$ cells were seeded in flat-bottom 6-well plates and incubated for $24 \mathrm{~h}$. The cells were then treated with Ad-LacZ and Ad-REIC at the indicated MOI in $0.5 \mathrm{ml}$ of complete medium for $1 \mathrm{~h}$, and $1.5 \mathrm{ml}$ of fresh medium was added and the cells were incubated for $72 \mathrm{~h}$. The apoptotic cells were visualized by Hoechst 33342 staining. Hoechst 33342 is an intercalating dye that can help to determine the total chromatin quantity variations and the degree of chromatin condensation $(18,19)$. The dye solution was added into the medium and incubated in the dark for $10 \mathrm{~min}$. The cells were directly observed with phase contrast and fluorescence microscopy. The apoptotic cells were identified by the presence of highly condensed or fragmented nuclei. Apoptotic cells were counted in five different fields using microscopic observations. One hundred cells were judged under one field.

Cell viability assay. The cells were detached by trypsin and $5.0 \times 10^{5}$ cells in $1 \mathrm{ml}$ complete medium were put into $15 \mathrm{ml}$ tube to assess the anti-proliferative effect of Ad-REIC in the floating cell condition. These floating cancer cells were treated with Ad-LacZ or with Ad-REIC at the indicated MOI with constant agitation for $1 \mathrm{~h}$. These cells were then seeded in flat-bottom 6 -well plates and incubated for $72 \mathrm{~h}$. The cell viability was determined using the MTS assay (CellTiter $96^{\circledR}$ Aqueous One Solution Cell Proliferation Assay, Promega Corp., Madison, WI, USA), according to the manufacturer's instructions.

The cells were seeded in flat-bottom 96-well microplates at a concentration of 1,000 cells per well to carry out the cell viability assay in the Ad-REIC and doxorubicin combined treatment. The cells were incubated for $24 \mathrm{~h}$ and treated with Ad-LacZ and Ad-REIC at $100 \mathrm{MOI}$ in the complete medium for $1 \mathrm{~h}$. The medium was exchanged to the fresh medium and the cells were incubated for $24 \mathrm{~h}$. The floating dead cells were removed and attached cells were treated with doxorubicin at the indicated concentration for $48 \mathrm{~h}$. The cell viability was determined using the MTS assay.

Western blot analysis. The cells were treated with Ad-LacZ and Ad-REIC at $100 \mathrm{MOI}$, and cultured for $24 \mathrm{~h}$. The floating dead cells were removed and attached cells were lysed for the sample. The cells were lysed with ice-cold lysis buffer to extract proteins. Insoluble fragments were removed by centrifugation, and the supernatants were adjusted to equal protein concentration in each experiment. Samples (10 $\mu \mathrm{g}$ of protein) were separated on a 7.5\% SDS-PAGE gel and transferred onto a polyvinylidene fluoride membrane (PVDF membranes; Millipore, Billerica, MA, USA) for western blotting. Following the transfer, the membranes were blocked for $1 \mathrm{~h}$ with $5 \%$ nonfat milk powder, $6 \%$ glycine and $0.1 \%$ Tween-20 in Tris buffered saline (TBS) at room temperature. The membranes were incubated for $1 \mathrm{~h}$ at room temperature with the primary antibodies; T5168 $(1: 8,000)$ for tubulin (Sigma), rabbit anti-human REIC/Dkk-3 antibody raised in this laboratory $(1: 1,000)$ and $\mathrm{C} 219(1: 250)$ for P-gp (Calbiochem, San Diego, CA, USA). After 3 washes in TBS supplemented with $0.1 \%$ Tween-20, the membranes were incubated with horseradish peroxidase-conjugated secondary antibody for $1 \mathrm{~h}$ at room temperature. The bound antibodies were visualized by the enhanced chemiluminescence detection method (ECL kit, Amersham Pharmacia Biotech, Chandler, AZ, USA) using medical X-ray film. JNK inhibitor $(1 \mu \mathrm{M})$ (SP600125, A.G. Scientific Inc., San Diego, CA, USA) was added to inhibit the kinase activity of JNK in some experiments.

Statistical analysis. The data are presented as the mean \pm SE. Student's unpaired t-test was performed for the statistical analysis between the two groups and the difference was considered to be significant at $p<0.05$. The analyses were carried out using the StatView 4.5 software package (Abacus Concepts, Berkeley, CA, USA). 
A
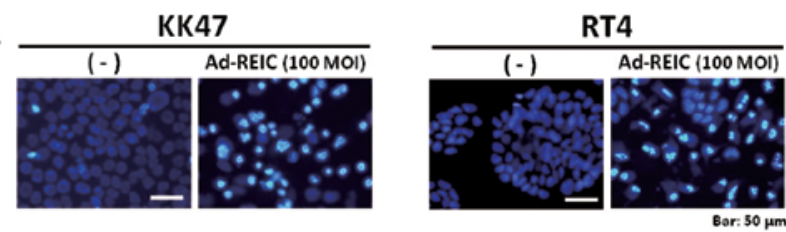

B

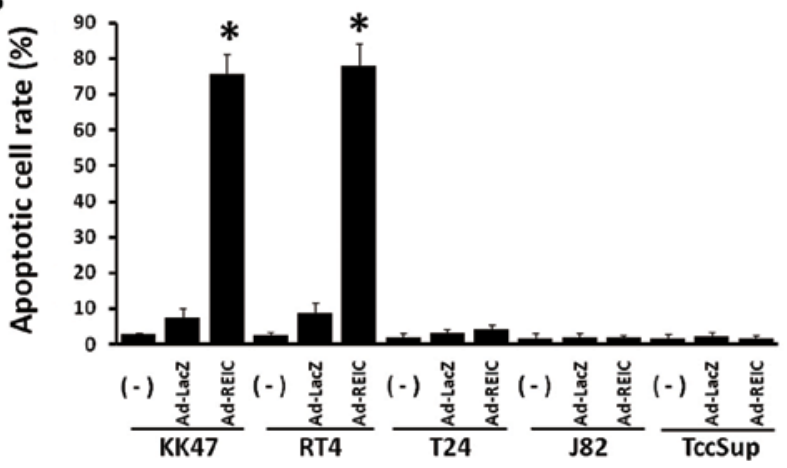

Figure 1. (A) Apoptosis induction after Ad-REIC treatment was analyzed by Hoechst 33342 staining in KK47 and RT4 human bladder cancer cells. Apoptotic cells were clearly observed as brightening cells under fluorescence microscopy. (B) The apoptotic cell rate was examined after the indicated treatment (no treatment, Ad-LacZ and Ad-REIC at $100 \mathrm{MOI}$ ) in various human bladder cancer cell lines (KK47, RT4, T24, J82, and TccSup). Apoptotic cells were counted by Hoechst 33342 staining. * A significant difference was observed between the control Ad-LacZ and Ad-REIC treatment.

\section{Results}

Apoptosis induction by Ad-REIC treatment in various human bladder cancer cell lines. Significant apoptotic induction was observed in KK47 and RT4 human bladder cancer cells after Ad-REIC treatment, but not in T24, J82 and TccSup cells (Fig. 1A and B). The incidence of apoptosis by Ad-REIC at $100 \mathrm{MOI}$ was $75.6 \%$ in KK47 and $78.0 \%$ in RT4 and a significant difference was observed in comparison to the control Ad-LacZ treatment at 100 MOI. The apoptotic cell rate by Ad-REIC in T24, J82 and TccSup human bladder cancer cells was $4.2 \%, 2.0 \%$ and $1.8 \%$, respectively. Therefore, KK47 and RT4 cells are sensitive to Ad-REIC treatment, however, T24, J82 and TccSup cells are resistant under these conditions.

Ad-REIC significantly reduces bladder cancer cell viability in the floating cell condition. T24, J82 and TccSup cancer cells were resistant to the Ad-REIC treatment for apoptosis induction under standard cell culture conditions. These resistant cancer cell lines were re-evaluated for Ad-REIC treatment in a floating cell condition, which is clinically observed after transurethral operation and becomes a cause of the intravesical cancer dissemination. The resistant cancer cell lines, T24 and J82 were treated with Ad-REIC at 100, 500 and 1,000 MOI in a floating culture for $1 \mathrm{~h}$ and then cultured for $72 \mathrm{~h}$ to access the anti-proliferative effect of Ad-REIC. Significant cell growth inhibition was observed in both T24 and J82 bladder cancer cell lines (Fig. 2A). The MTS assay showed that the cell viability at Ad-REIC 1,000 MOI was $20.0 \%$ in T24 and $18.2 \%$ in J82 cells and a significant reduction in cell viability was observed in comparison to that of the control Ad-LacZ treatment at 1,000 MOI (Fig. 2B). Therefore, significant cell growth inhibition was observed in T 24 and $\mathbf{J} 28$ cells by the floating cell culture
A

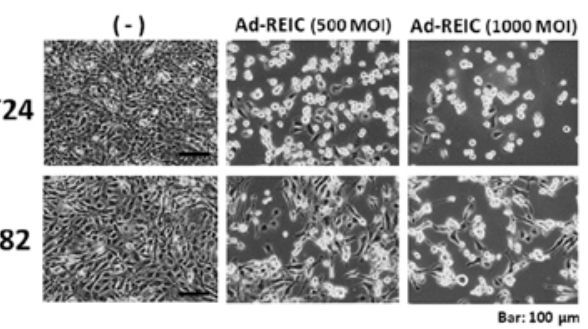

B

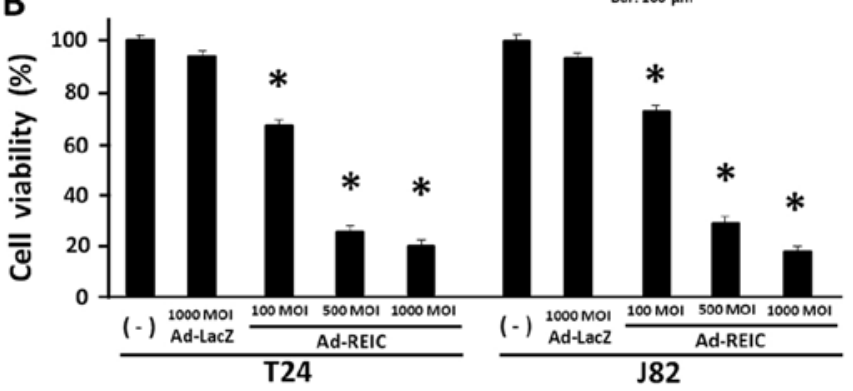

Figure 2. (A) The Ad-REIC resistant cancer cell lines (T24 and J82) in Fig. 1 were treated with Ad-REIC in floating cell conditions. The floating cancer cells were treated with Ad-REIC at the indicated MOI for $1 \mathrm{~h}$ with constant agitation. After the treatment, the cells were seeded in flat-bottom 6-well plates and incubated for $72 \mathrm{~h}$. The representative microscopic image in each treatment is shown. (B) The floating T24 and J82 cancer cells were treated with the control (no treatment and Ad-LacZ at 1,000 MOI) and Ad-REIC treatment at 100, 500 and 1,000 MOI. The cells were seeded in flat-bottom 6-well plates and incubated for $72 \mathrm{~h}$. The cell viability is then examined by MTS assay in the T24 and J82 cancer cells. * A significant difference was observed between the control Ad-LacZ and Ad-REIC treatment. The cell viability of no treatment was considered $100 \%$ and the relative cell viability was calculated in the indicated treatment.

with Ad-REIC at 100, 500 and 1,000 MOI. The TccSup cancer cells also showed significant growth suppression following the Ad-REIC treatment at 1,000 MOI in comparison to the control Ad-LacZ treatment (data not shown).

Apoptosis induction in KK47/Wt and KK47/ADM cells by Ad-REIC treatment. Apoptosis induction by Ad-REIC was assayed in adriamycin resistant KK47 bladder cancer cells (KK47/ADM) which also presents multidrug resistance (16). The incidence of apoptosis by Ad-REIC at $100 \mathrm{MOI}$ was $75.6 \%$ in KK47/Wt and 65.2\% in KK47/ADM and significant apoptosis induction was observed in comparison to the control Ad-LacZ treatment (Fig. 3). The incidence of apoptosis by Ad-REIC at $10 \mathrm{MOI}$ was not significant in either KK47/Wt or KK47/ADM cells in comparison to the control Ad-LacZ treatment.

Ad-REIC treatment sensitizes multidrug resistant KK47/ADM cells to doxorubicin. The multidrug resistant KK47/ADM cells were used to determine the effect of combined treatment with Ad-REIC and doxorubicin (adriamycin). KK47/Wt and KK47/ ADM cells were divided into three groups of no treatment, Ad-LacZ and Ad-REIC at 100 MOI. The ability of Ad-REIC to reverse drug resistance was evaluated by exposing the cells to increasing concentrations of doxorubicin. KK47/Wt cells showed no significant difference between the treatment groups (Fig. 4). However, Ad-REIC treatment significantly shifted the doseresponse curve for doxorubicin toxicity to lower concentrations in KK47/ADM cells (Fig. 4). The cell viability at $2.5 \mu \mathrm{M}$ doxorubicin was $80.4 \%, 85.3 \%$ and $42.3 \%$ in the no treatment, Ad-LacZ 


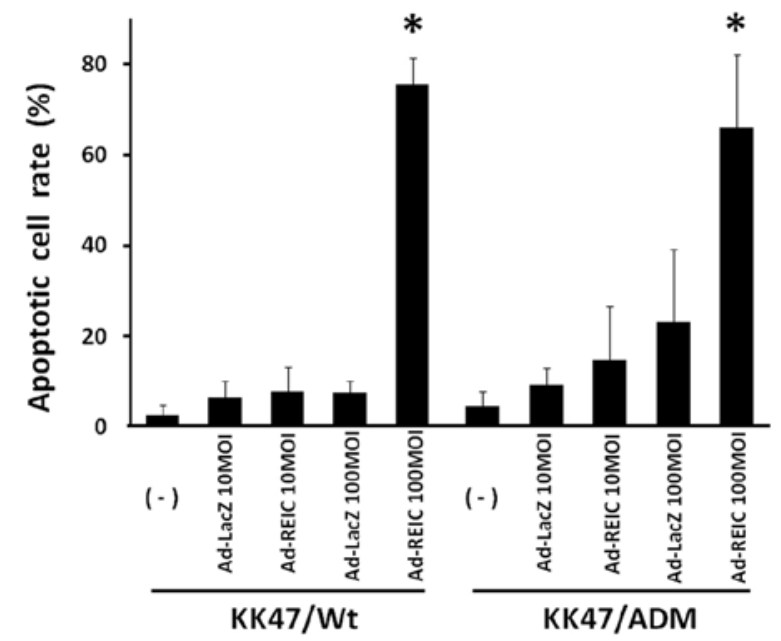

Figure 3. KK47/Wt and multidrug resistant KK47/ADM bladder cancer cells were treated with Ad-REIC at the indicated MOI. Apoptotic cells were counted by Hoechst 33342 staining. * A significant difference was observed in comparison to the control Ad-LacZ at $100 \mathrm{MOI}$.
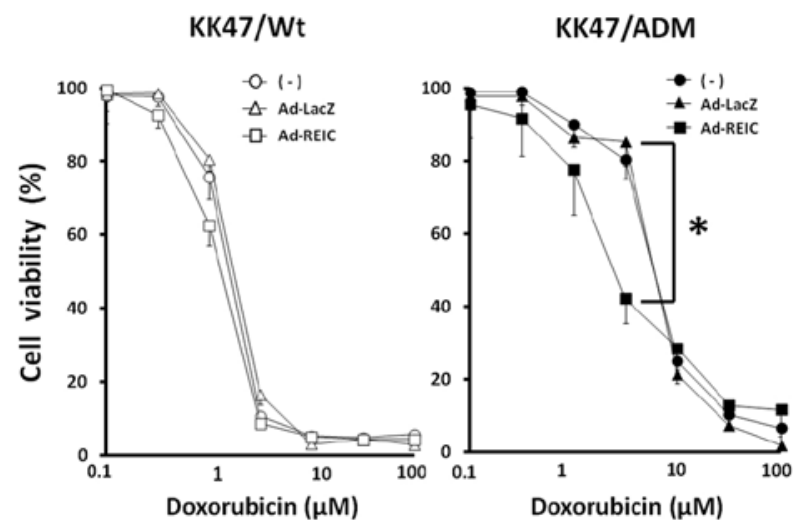

Figure 4. The sensitivity of multidrug resistant KK47/ADM bladder cancer cells to doxorubicin is restored by Ad-REIC treatment. KK47/Wt and KK47/ADM cells were treated (no treatment, Ad-LacZ and Ad-REIC at $100 \mathrm{MOI}$ ), and then incubated for $48 \mathrm{~h}$ in various concentrations of doxorubicin. The dose-response curves against doxorubicin toxicity are shown based on the cell viability assay. KK47/Wt cells show similar dose-response curves for doxorubicin in each treatment group. * A significant difference was observed in KK47/ADM cells between Ad-LacZ and Ad-REIC at $2.5 \mu \mathrm{M}$ doxorubicin. The cell viability at $0 \mu \mathrm{M}$ doxorubicin was considered $100 \%$ in each treatment and the relative cell viability was calculated at the indicated concentration.

and Ad-REIC groups, respectively. Therefore, Ad-REIC treatment partially restored the sensitivity of KK47/ADM cells to doxorubicin.

Ad-REIC treatment downregulates $P$-gp expression in KK47/ ADM cells in a JNK-dependent manner. The expression level of REIC/Dkk-3 and P-gp after Ad-REIC treatment was examined using western blot analysis (Fig. 5). REIC/Dkk-3 protein was strongly expressed by Ad-REIC treatment in both KK47/Wt and KK47/ADM cells. P-gp, the representative multidrug resistant protein, was expressed in KK47/ADM cells with no treatment or Ad-LacZ treatment. Ad-REIC treatment significantly downregulated the expression of P-gp in KK47/ADM cells. The JNK pathway is a crucial factor for the cancer specific apoptosis in

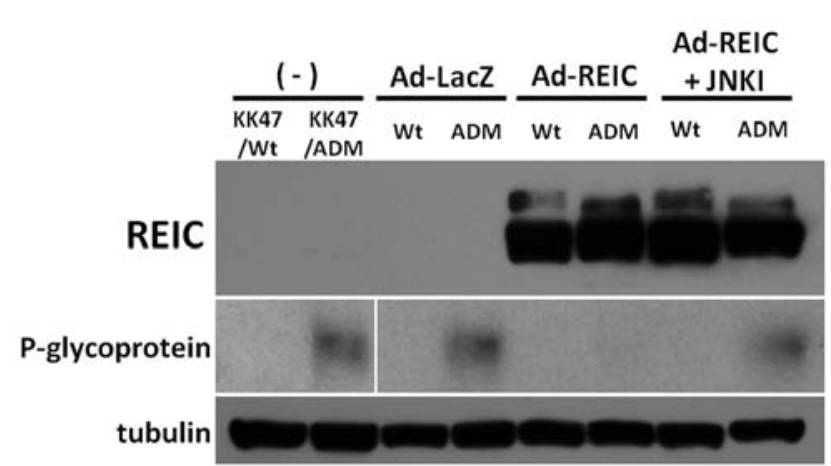

Figure 5. The expression of P-glycoprotein in multidrug resistant KK47/ ADM bladder cancer cells is down-regulated by Ad-REIC treatment in a JNK-dependent manner. KK47/Wt and KK47/ADM cells were treated with Ad-LacZ and Ad-REIC at 100 MOI. The expression levels of REIC/Dkk-3 protein and P-glycoprotein were determined by western blot analysis. Tubulin was used as a loading control. A JNK inhibitor (JNKI), SP600125 was used to inhibit the kinase activity of JNK.

Ad-REIC treatment (5). SP600125, a JNK inhibitor, inhibits the kinase activity of JNK (20). Combined treatments with Ad-REIC and SP600125 reversed the expression level of P-gp in KK47/ ADM cells. Therefore, Ad-REIC treatment suppressed the P-gp expression in the multidrug resistant KK47/ADM cells in a JNKdependent manner.

\section{Discussion}

This study showed that KK47 and RT4 cells are sensitive to Ad-REIC treatment for apoptosis induction and three human bladder cancer cell lines, T24, J82, and TccSup, are resistant. However, the efficacy of Ad-REIC treatment must not be evaluated in the standard cell culture conditions but in floating cell conditions in order to demonstrate the utility of Ad-REIC as intravesical therapeutic agent to prevent the recurrence of bladder cancer. Floating cancer cells are clinically observed after transurethral operation and are a cause of the intravesical cancer dissemination and recurrence. We herein demonstrated that Ad-REIC treatment significantly inhibited cell proliferation of the resistant bladder cancer cell lines in the floating cell condition. Since the Ad-REIC treatment did not inhibit proliferation of T24 and J82 cancer cells under the standard cell culture condition (data not shown), the floating cancer cells are more sensitive to Ad-REIC treatment. It is conceivable that Ad-REIC could transfect more efficiently to the floating cancer cells than the attached cells on the dish and therefore indicated the significant anti-cancer effect in the floating cell condition. The influence of adenovirus itself on the cells can be denied, because there was no significant difference between no treatment and Ad-LacZ treatment. Finally, Ad-REIC has anti-cancer effect in 6 human bladder cancer cell lines, RT4, KK47/Wt, KK47/ADM, T24, J82 and TccSup. This result suggests that the intravesical instillation with Ad-REIC could be an attractive therapeutic strategy to treat superficial lesion and floating/disseminated cells of human bladder cancer.

Bladder cancer often recurs after the transurethral resection of bladder tumors and such recurrent tumors arise at different sites in the urothelium. Even though the tumor is completely 
resected macroscopically by the operation, microscopic floating or disseminated cells will implant in the bladder epithelium and the spread of the original clone forms multifocal tumors (21). The intravesical instillation of chemotherapeutic agents immediately after transurethral operation is clinically performed to prevent tumor recurrence $(22,23)$ and it is important for the intravesical chemotherapy to kill the floating or disseminated malignant cells in the bladder. Representative anti-cancer agents for intravesical chemotherapy are epirubicin, mitomycin $\mathrm{C}$ and doxorubicin. However, multidrug resistant cancer cells or multidrug resistant cancer cells newly emerge during repeated doxorubicin treatments. The present study showed that combination therapy with Ad-REIC and doxorubicin significantly suppressed the growth of the multidrug resistant cell line KK47/ADM by restoring the sensitivity to doxorubicin.

Multidrug resistance is mainly attributed to the overexpression of efflux transporters such as P-gp and MRP1. P-gp and MRP1 are members of the ATP binding cassette (ABC) superfamily of transporters and are capable of effluxing many chemotherapeutics out of cancer cells, and allowing them to survive the toxic insult $(13,14)$. There is a positive correlation between the expression of P-gp and multidrug resistant phenotypes in transitional cell carcinoma (24). On the other hand, the relationship between P-gp expression and JNK pathway was highlighted in a previous study showing that enhancement of the JNK pathway downregulates P-gp and reverses $\mathrm{P}$-gp mediated multidrug resistance in cancer cells (15). Overexpression of REIC/Dkk-3 by Ad-REIC in cancer cells gives rise to endoplasmic stress and induces cancer cell specific apoptosis through the activation of $\mathrm{JNK}$ and c-Jun, whereas apoptosis is not induced in normal cells $(5,25)$. The Ad-REIC treatment in KK47/ADM cells seemed to downregulate P-gp expression through JNK activation and reversed the drug resistance to doxorubicin. Similar findings were also obtained in the multidrug resistant human breast cancer MCF7/ADR cells. Ad-REIC treatment upregulates the expression of phosphorylated JNK and c-Jun in MCF7/ADR cells, and downregulates the level of P-gp following partial reversal of doxorubicin resistance (26).

The current study demonstrated two therapeutic aspects of the Ad-REIC agent against human bladder cancer cells, namely as an apoptosis inducer/cell growth inhibitor and as a sensitizer to chemotherapeutic agents in the multidrug resistant cancer cells. Therefore, the intravesical instillation of Ad-REIC could be an attractive therapeutic method in human bladder cancer where the treatment of superficial lesions and floating/disseminated or multidrug resistant cancer cells is required.

\section{References}

1. Dobruch J and Herr H: Should all patients receive single chemotherapeutic agent instillation after bladder tumour resection? BJU Int 104: 170-174, 2009.

2. Kurth KH, Bouffioux C, Sylvester R, van der Meijden AP, Oosterlinck W and Brausi M: Treatment of superficial bladder tumors: achievements and needs. The EORTC Genitourinary Group. Eur Urol 37 (Suppl 3): 1-9, 2000.

3. Tsuji T, Miyazaki M, Sakaguchi M, Inoue Y and Namba M A REIC gene shows down-regulation in human immortalized cells and human tumor-derived cell lines. Biochem Biophys Res Commun 268: 20-24, 2000.
4. Tsuji T, Nozaki I, Miyazaki M, Sakaguchi M, Pu H, Hamazaki Y, Iijima $\mathrm{O}$ and Namba M: Antiproliferative activity of REIC/Dkk-3 and its significant down-regulation in non-small-cell lung carcinomas. Biochem Biophys Res Commun 289: 257-263, 2001.

5. Abarzua F, Sakaguchi M, Takaishi M, Nasu Y, Kurose K, Ebara S, Miyazaki M, Namba M, Kumon H and Huh NH: Adenovirusmediated overexpression of REIC/Dkk-3 selectively induces apoptosis in human prostate cancer cells through activation of c-Jun-NH2-kinase. Cancer Res 65: 9617-9622, 2005.

6. Abarzua F, Sakaguchi M, Tanimoto R, Sonegawa H, Li DW Edamura K, Kobayashi T, Watanabe M, Kashiwakura Y, Kaku H, Saika T, Nakamura K, Nasu Y, Kumon H and Huh NH: Heat shock proteins play a crucial role in tumor-specific apoptosis by REIC/Dkk-3. Int J Mol Med 20: 37-43, 2007.

7. Edamura K, Nasu Y, Takaishi M, Kobayashi T, Abarzua F, Sakaguchi M, Kashiwakura Y, Ebara S, Saika T, Watanabe M, Huh NH and Kumon H: Adenovirus-mediated REIC/Dkk-3 gene transfer inhibits tumor growth and metastasis in an orthotopic prostate cancer model. Cancer Gene Ther 14: 765-772, 2007.

8. Watanabe M, Kashiwakura Y, Huang P, Ochiai K, Futami J, Li SA, Takaoka M, Nasu Y, Sakaguchi M, Huh NH and Kumon H: Immunological aspects of REIC/Dkk-3 in monocyte differentiation and tumor regression. Int J Oncol 34: 657-663, 2009.

9. Kashiwakura Y,Ochiai K, Watanabe M, Abarzua F, Sakaguchi M, Takaoka M, Tanimoto R, Nasu Y, Huh NH and Kumon H: Downregulation of inhibition of differentiation-1 via activation of activating transcription factor 3 and Smad regulates REIC/ Dickkopf-3-induced apoptosis. Cancer Res 68: 8333-8341, 2008.

10. Tanimoto R, Abarzua F, Sakaguchi M, Takaishi M, Nasu Y, Kumon H and Huh NH: REIC/Dkk-3 as a potential gene therapeutic agent against human testicular cancer. Int J Mol Med 19: 363-368, 2007.

11. Jin Y, Murata H, Sakaguchi M, Kataoka K, Watanabe M, Nasu Y, Kumon $\mathrm{H}$ and Huh NH: Partial sensitization of human bladder cancer cells to a gene-therapeutic adenovirus carrying REIC/ Dkk-3 by downregulation of BRPK/PINK1. Oncol Rep 27: 695-699, 2012.

12. Zincke H, Utz DC, Taylor WF, Myers RP and Leary FJ: Influence of thiotepa and doxorubicin instillation at time of transurethral surgical treatment of bladder cancer on tumor recurrence: a prospective randomized, double blind, controlled trial. J Urol 129: 505-509, 1983.

13. Cole SP, Bhardwaj G, Gerlach JH, Mackie JE, Grant CE, Almquist KC, Stewart AJ, Kurz EU, Duncan AM and Deeley RG: Overexpression of a transporter gene in a multidrug-resistant human lung cancer cell line. Science 258: 1650-1654, 1992.

14. Gottesman MM and Pastan I: Biochemistry of multidrug resistance mediated by the multidrug transporter. Annu Rev Biochem 62: 385-427, 1993.

15. Zhou J, Liu M, Aneja R, Chandra R, Lage H and Joshi HC: Reversal of P-glycoprotein-mediated multidrug resistance in cancer cells by the c-Jun NH2-terminal kinase. Cancer Res 66: 445-452, 2006

16. Kimiya K, Naito S, Soejima T, Sakamoto N, Kotoh S, Kumazawa J and Tsuruo T: Establishment and characterization of doxorubicinresistant human bladder cancer cell line, KK47/ADM. J Urol 148: 441-445, 1992.

17. Abarzua F, Kashiwakura Y, Takaoka M, Watanabe M, Ochiai K Sakaguchi M, Iwawaki T, Tanimoto R, Nasu Y, Huh NH and Kumon H: An N-terminal 78 amino acid truncation of REIC/ Dkk-3 effectively induces apoptosis. Biochem Biophys Res Commun 375: 614-618, 2008.

18. Belloc F, Dumain P, Boisseau MR, Jalloustre C, Reiffers J, Bernard P and Lacombe F: A flow cytometric method using Hoechst 33342 and propidium iodide for simultaneous cell cycle analysis and apoptosis determination in unfixed cells. Cytometry 17: 59-65, 1994.

19. Maciorowski Z, Delic J, Padoy E, Klijanienko J, Dubray B, Cosset JM, Dumont J, Magdelénat $\mathrm{H}$ and Vielh P: Comparative analysis of apoptosis measured by Hoechst and flow cytometry in non-Hodgkin's lymphomas. Cytometry 32: 44-50, 1998.

20. Bennett BL, Sasaki DT, Murray BW, O'Leary EC, Sakata ST, $\mathrm{Xu}$ W, Leisten JC, Motiwala A, Pierce S, Satoh Y, Bhagwat SS, Manning AM and Anderson DW: SP600125, an anthrapyrazolone inhibitor of Jun N-terminal kinase. Proc Natl Acad Sci USA 98: 13681-13686, 2001.

21. Harris AL and Neal DE: Bladder cancer - field versus clonal origin. N Engl J Med 326: 759-761, 1992. 
22. Oosterlinck W, Kurth KH, Schroder F, Bultinck J, Hammond B and Sylvester R: A prospective European Organization for Research and Treatment of Cancer Genitourinary Group randomized trial comparing transurethral resection followed by a single intravesical instillation of epirubicin or water in single stage Ta, T1 papillary carcinoma of the bladder. J Urol 149: 749-752, 1993

23. Solsona E, Iborra I, Ricos JV, Monros JL, Casanova J and Dumont R: Effectiveness of a single immediate mitomycin $\mathrm{C}$ instillation in patients with low risk superficial bladder cancer: short and long-term follow-up. J Urol 161: 1120-1123, 1999.

24. Naito S, Sakamoto N, Kotoh S, Goto K, Matsumoto T and Kumazawa J: Correlation between the expression of P-glycoprotein and multidrug-resistant phenotype in transitional cell carcinoma of the urinary tract. Eur Urol 22: 158-162, 1992.
25. Sakaguchi M, Kataoka K, Abarzua F, Tanimoto R, Watanabe M, Murata H, Than SS, Kurose K, Kashiwakura Y, Ochiai K, Nasu Y, Kumon H and Huh NH: Overexpression of REIC/Dkk-3 in normal fibroblasts suppresses tumor growth via induction of interleukin-7. J Biol Chem 284: 14236-14244, 2009.

26. Kawasaki K, Watanabe M, Sakaguchi M, Ogasawara Y, Ochiai K, Nasu Y, Doihara H, Kashiwakura Y, Huh NH, Kumon H and Date H: REIC/Dkk-3 overexpression downregulates P-glycoprotein in multidrug-resistant MCF7/ADR cells and induces apoptosis in breast cancer. Cancer Gene Ther 16: 65-72, 2009. 